\section{SLC01B1 variants associated with statin-induced myopathy}

Statin therapy has proven efficacy for reducing the incidence of major adverse clinical events, but when administered in high doses these agents can cause myopathy. A means to identify those at increased risk of statin-induced myopathy could improve these agents' safety profiles and enable individualized dosing. The SEARCH investigators performed a wellpowered, genomewide association study and identified common single-nucleotide polymorphisms (SNPs) that are strongly associated with statin-related myopathy.

The original SEARCH trial randomly allocated 6,031 patients to receive $80 \mathrm{mg}$ simvastatin, of whom 85 developed myopathy and were genotyped for this study. The only association between myopathy and a SNP to yield an uncorrected $P$ value of $<10^{-5}$ was that between myopathy and the rs 4363657 variant, a noncoding SNP of SLCO1B1 $(P=0.001$ with the Bonferroni correction). Of note, SLCO1B1 encodes OATP1B1, a protein that regulates uptake of statins into the liver. Of the additional SNPs within SLCO1B1 and flanking regions that were identified, only one-rs4149056-was both in nearly complete linkage disequilibrium with rs4363657 and nonsynonymous. Odds ratios for myopathy were 4.5 per copy of the $\mathrm{C}$ allele $(95 \% \mathrm{Cl}$ 2.6-7.7) and 16.9 for CC homozygotes (95\% Cl 4.7-61.1), when compared with $\Pi$ homozygotes $\left(P=2 \times 10^{-9}\right)$. Among the 90 control patients who also received highdose simvastatin, the population prevalence of rs 4149056 was 0.15 . Importantly, the association between rs4149056 and myopathy was validated by data from the HPS $(P=0.004$; relative risk per $\mathrm{C}$ allele copy $2.6,95 \% \mathrm{Cl} 1.3-5.0$ ).

Original article The SEARCH Collaborative Group (2008)

SLCO1B1 variants and statin-induced myopathya genomewide study. N Engl J Med 359: 789-799

\section{Cardiac MRI reveals the clinical importance of LV mass in hypertrophic cardiomyopathy}

Hypertrophic cardiomyopathy (HCM) has a heterogeneous phenotype that is not easily defined by two-dimensional echocardiography alone. In this multicenter study, Olivotto et al. used cardiac MRI to measure and compare the left ventricular (LV) dimensions of 606 healthy individuals with those of 264 patients with $\mathrm{HCM}$. Their investigation provides new insights into the clinical correlates and importance of LV mass in patients with this condition.

In general, individuals with HCM had a higher LV mass index than healthy participants $\left(104 \pm 40 \mathrm{~g} / \mathrm{m}^{2}\right.$ vs $61 \pm 10 \mathrm{~g} / \mathrm{m}^{2}$ in men and $89 \pm 33 \mathrm{~g} / \mathrm{m}^{2}$ vs $47 \pm 7 \mathrm{~g} / \mathrm{m}^{2}$ in women; $P<0.0001$ for both). Notably, however, LV mass index was within the normal range in $20 \%$ of patients with $\mathrm{HCM}$ and only moderately elevated in $16 \%$. Multivariate analysis revealed that male sex and outflow obstruction were independent predictors of increased LV mass index $(P<0.001$ for both). Increased maximal wall thickness and increased LV mass index were weakly, but significantly, correlated. Patients who died from HCM had a higher mean LV mass index than those who survived $(P=0.02)$. Sensitivity for prediction of HCM-related mortality was $100 \%$ for increased LV mass index $\left(>91 \mathrm{~g} / \mathrm{m}^{2}\right.$ in men and $>69 \mathrm{~g} / \mathrm{m}^{2}$ in women) and $90 \%$ for maximal LV wall thickness $>30 \mathrm{~mm}$. The authors suggest that, contrary to popular belief, the presence of increased LV mass is not required for a diagnosis of $\mathrm{HCM}$ to be made.

\section{Original article Olivotto I et al. (2008) Assessment and significance of left ventricular mass by cardiovascular magnetic resonance in hypertrophic cardiomyopathy. J Am Coll Cardiol 52: 559-566 \\ Correlations between different atherogenic indices are altered by statins}

Apolipoprotein B concentration is the strongest lipid-associated predictor of cardiovascular events. However, in patients who require lipidlowering therapy, LDL cholesterol is the primary therapeutic target; non-HDL cholesterol is recommended as a secondary target for patients with elevated triglyceride levels.

Statin-associated reductions in LDLcholesterol levels are known to be disproportionate to reductions in apolipoprotein $B$ levels. Ballantyne and colleagues, therefore, studied the relationship between apolipoprotein $B$ concentrations and levels of LDL cholesterol and non-HDL cholesterol in patients before and after initiation of statin therapy.

The ACC-recommended therapeutic targets are apolipoprotein $B<0.9 \mathrm{~g} / \mathrm{l}$, LDL 